\title{
A new method of assessment and equations on Carbon footprint
}

\author{
Sameer Sawant* ${ }^{1,2}$ and Basavraj Babaleshwar ${ }^{1,2}$ \\ ${ }^{I}$ CEFA group, Talegaon Dabhade, Pune, India \\ ${ }^{2}$ Sir Parshurambhau College, Pune, India
}

\begin{abstract}
Carbon footprint or green terminology has become famous over a few decades in many countries including India. Several industrial development schemes and policies are developed and are developing which broadly focuses on green terminology [1,2]. Although the terms such as carbon footprint, ecological footprint and green terminology are much popular, there exists no standard ideology or a theory accepted so far which would be used worldwide and thereby ends this long lasting debate. This article focuses on a new theory developed in order to minimize the problems, uncertainties and errors related to the previous studies and to attain precise completeness to the assessment with the help of equations which would also lead further research and development in the field.
\end{abstract}

Keywords: Carbon footprint, ecological footprint, green terminology, emission factor, carbon intensity, work done.

\section{Introduction}

Carbon dioxide $\left(\mathrm{CO}_{2}\right)$ is one of the prominent Greenhouse-gases (GHGs) responsible for global warming. Along with $\mathrm{CO}_{2}$ there are other GHGs like $\mathrm{CH}_{4}, \mathrm{~N}_{2} \mathrm{O}, \mathrm{HFCs}$, PFCs and $\mathrm{SF}_{6}[4,6]$ which are enlisted in the Kyoto protocol [6] which add to greenhouse effects and global warming. GHGs are emitted through various natural processes and human activities. Natural processes include volcanic eruptions, forest fires, earthquakes, etc. Human activities include excessive usage of fuel, improper methods of waste disposal, rapid growth in industrialization and urbanization, improper sewage practices, etc. Emission of GHGs in the atmosphere leads to increase in the rate of global warming. The consequences of global warming are harsh. Floods, climate changes, draughts, untimely rains, forest fires etc. all are the consequences of global warming which affect human life directly or indirectly and also may lead to death. Though emission of GHGs through natural processes cannot be controlled, the emission through human activities can be evaluated and corrective measures can be implemented in order to control the rate of GHG emissions. Although it's presumed that global warming can't be stopped, but the rate at which it occurs can be controlled. There is necessity of proper method based upon precise and exclusive definitions and equations for understanding and evaluating carbon footprint more precisely and scientifically which would help further practical research and development.

This article focuses on precise methodology for evaluation of carbon footprint and conveys new definitions, thus resulting new equations for corrective data analysis. It also includes a case study of Sir Parshurambhau College, Pune, India (the campus area is 25 acres) for verification and clarity of the proposed methodology.

\section{Brief Review}

The concept of carbon footprint derives from the concept of ecological footprint raised by Wackernagel and Rees in 1996 [17]. As one part of Ecological Footprint, the land area needed to sequester $\mathrm{CO}_{2}$ emitted from burning fossil fuel is measured to estimate the land requirement for energy use [17]. There exist several definitions on carbon footprint in wider literature. Yet no academic definition has been provided which would be widely accepted.

A definition with practical solution is said to be proposed by Wiedmann and Minx: "The carbon footprint is a measure of the exclusive total amount of carbon dioxide emissions that is directly or indirectly caused by an activity or is accumulated over the life stages of a product." According to this definition, exclusively only $\mathrm{CO}_{2}$ emissions must be included in order to account carbon footprint; presuming the fact that it is difficult to quantify other substances with green house warming potential. In contradiction, DEFRA has successfully evaluated emission factors of other substances $\left(\mathrm{CH}_{4}\right.$ and $\left.\mathrm{N}_{2} \mathrm{O}\right)$ for various inventories and furthermore expressed emission factors in carbon dioxide equivalents $\left(\mathrm{CO}_{2} \mathrm{e}\right)$. The definition also refrains from expressing carbon footprint as area based indicator $[3,8]$ in order to avoid conversions to an area unit (ha, $\mathrm{m}^{2}$, $\mathrm{km}^{2}$, etc). Along with methodological issues, it also involves carbon footprint comparison (between various functional units) issue and errors since it is expressed in mere mass units ( $\mathrm{kg}, \mathrm{t}$, etc). While righteously, the authors hold to inclusion of indirect emissions in the evaluation of carbon footprint. It has been demonstrated by some case studies that indirect emissions constitute the majority of carbon footprint of a functional unit [16]. 
An organizational definition of carbon footprint is provided by Tao Gao, Qing Liu and Jianping Wang: "An organizational carbon footprint measures the GHG emissions from all activities across the organization including energy used in buildings, industrial processes and company vehicles." In this definition, other substances or GHGs including $\mathrm{CO}_{2}$ are included for rightful carbon footprint assessment. A terminal consumption analysis method based on the IO analysis is used as a method for evaluating organizational carbon footprint [14]. But, similar to the definition provided by Wiedmann and Minx this definition too lacks the element for comparison between different scale functional units.

For calculation of carbon footprint of a mega structure such as city or a municipality the following definition is proposed by Larsen and Hertwich: "Carbon footprint is the life-cycle GHG emissions caused by the production of goods and services consumed by a geographically-defined population or activity, independent of whether the GHG emissions occur inside or outside the geographical borders of the population or activity of interest." [15] According to this definition carbon footprint refers to GHG emissions based on the consumption of a defined population, therefore calculation of the carbon footprint of a city or municipality should not be limited to its geopolitical boundaries [16]. It provides an element for comparison between cities by promoting evaluation of carbon footprint as GHG emissions per-capita. But population in a geopolitical area or a city is variable or can be said to be constantly increasing and therefore this may introduce uncertainties and fatal errors in setting GHG mitigation goals and comparing its own yearly carbon footprints.

All the above definitions involve methodological issues, evaluation and comparison errors or incompleteness. For precise evaluation and exhaustive study of carbon footprint patterns, spatial and temporal boundaries are necessary to be set and must be included in an ideal definition of carbon footprint. Such a definition has been proposed by Glen Peters: "The 'carbon footprint' of a functional unit is the climate impact under a specified metric that considers all relevant emission sources, sinks, and storage in both consumption and production within the specified spatial and temporal system boundary." Following the definition of a carbon footprint is the notion of 'embodied carbon', 'carbon flows', 'embedded carbon', 'virtual carbon', and similar terms. Historically, the emissions that occur along the supply chain of a functional unit have been said to be 'emissions embodied' in the functional unit. The emissions are not a physical part of the functional unit, but are associated with the functional unit via the production network [13]. Although the definition seems to be of a wider and open perspective, it involves other necessary and prominent terms such as sinks and storage which couldn't be ignored. Inclusion of spatial and temporal boundaries adds preciseness to the evaluation of carbon footprint and to the further study. The method to determine carbon footprint is not included in the definition which adds methodological issue.

\section{New Theory}

We propose the following definition, eliminating methodological confusions and minimizing errors and uncertainties.

A carbon footprint of a functional unit is a measure of 'work done' (expressed in kWyear) by the GHG emissions (expressed as $\mathrm{CO}_{2} \mathrm{e}$ ) considering all relevant sources, sinks and storage in both consumption and production within specified spatial and temporal system boundary.

This definition we proposed is similar to that proposed by Peters. As said above, the definition of Peters is precise and ideal, except there is no method of determination of carbon footprint provided. We included evaluation of work done by the GHG emissions in the definition, making it more precise. Work done is expressed in joules and it provides more scientific information about GHG emissions. The evaluation of work done is necessary and the equations required for this are discussed further including an example of a case study. The very first stage of carbon footprint assessment is data collection. The four stages of data collection we used are

a) Dividing the institute into discrete departments

b) Finding and listing the sources of emissions from every department

c) Preparing exhaustive questionnaire for every department separately

d) Data gathering and data extrapolation wherever inadequate data are available

Once the data are available, we need to evaluate the mass of carbon dioxide equivalents (tCO2e) as activity data (AD) times the standard emission factor (EF) (emission factors as shown in table 1)

$$
\mathrm{AD} \times \mathrm{EF}=\mathrm{tCO}_{2} \mathrm{e}
$$


It's presumed that evaluation of mass of $\mathrm{CO}_{2} \mathrm{e}$ will introduce some uncertainties because of data extrapolation. Also, the use of standard emission factors may introduce some error on the data because it's known that the emission rates may vary according to the region and locality. As far as possible authentic data must be gathered avoiding extrapolation [19] and if possible, the correct emission factors for the region or locality must be found to avoid errors.

Table 1: Emission factors for conversion (The emission factors are standard and taken from - Carbon gas conversion factor respiratory- DEFRA [7])

\begin{tabular}{|l|l|l|l|}
\hline Sr. No. & Inventories & Emission Factors & Units \\
\hline & & & \\
\hline 1 & Human & 1.1 & $\mathrm{kgCO}_{2} \mathrm{e} / 24 \mathrm{hr}$ \\
\hline 2 & Petrol & 2.1914 & $\mathrm{kgCO}_{2} \mathrm{e} / 1$ \\
\hline 3 & Diesel & 2.6024 & $\mathrm{kgCO}_{2} \mathrm{e} / 1$ \\
\hline 5 & Water supply & 0.0014 & $\mathrm{kgCO}_{2} \mathrm{e} / \mathrm{l}$ \\
\hline 6 & Paper & 0.928 & $\mathrm{kgCO}_{2} \mathrm{e} / \mathrm{kg}$ \\
\hline 7 & Solid waste & 0.021 & $\mathrm{kgCO}_{2} \mathrm{e} / \mathrm{kg}$ \\
\hline 8 & LPG & 0.214 & $\mathrm{kgCO}_{2} \mathrm{e} / \mathrm{kWh}$ \\
\hline 9 & Electricity & 0.856 & $\mathrm{kgCO}_{2} \mathrm{e} / \mathrm{kWh}$ \\
\hline 10 & Plastic & 2.154 & $\mathrm{kgCO}_{2} \mathrm{e} / \mathrm{kg}$ \\
\hline & Food and drinks & 3.59 & $\mathrm{kgCO}_{2} \mathrm{e} / \mathrm{kg}$ \\
\hline
\end{tabular}

We evaluate $\mathrm{kgCO}_{2} \mathrm{e}$ from all inventories responsible for GHG emissions using the above standard conversion factors [7, 11] as shown in Table 2 below. Although some extrapolation has been accounted (wherever utmost necessary), most of the data are authentic and provided by the valid authorities of the respective departments. For example, non-hostel (other) students are presumed to spend six hours in the campus. Diesel vehicles are presumed to travel $4.7 \mathrm{~km} / \mathrm{l}$ [12], whereas petrol vehicles are presumed to travel $18.8 \mathrm{~km} / \mathrm{l}$.

Table 2: Found emission sources for Sir Parshurambhau College and their evaluation in $\mathrm{kgCO}_{2} \mathrm{e}$

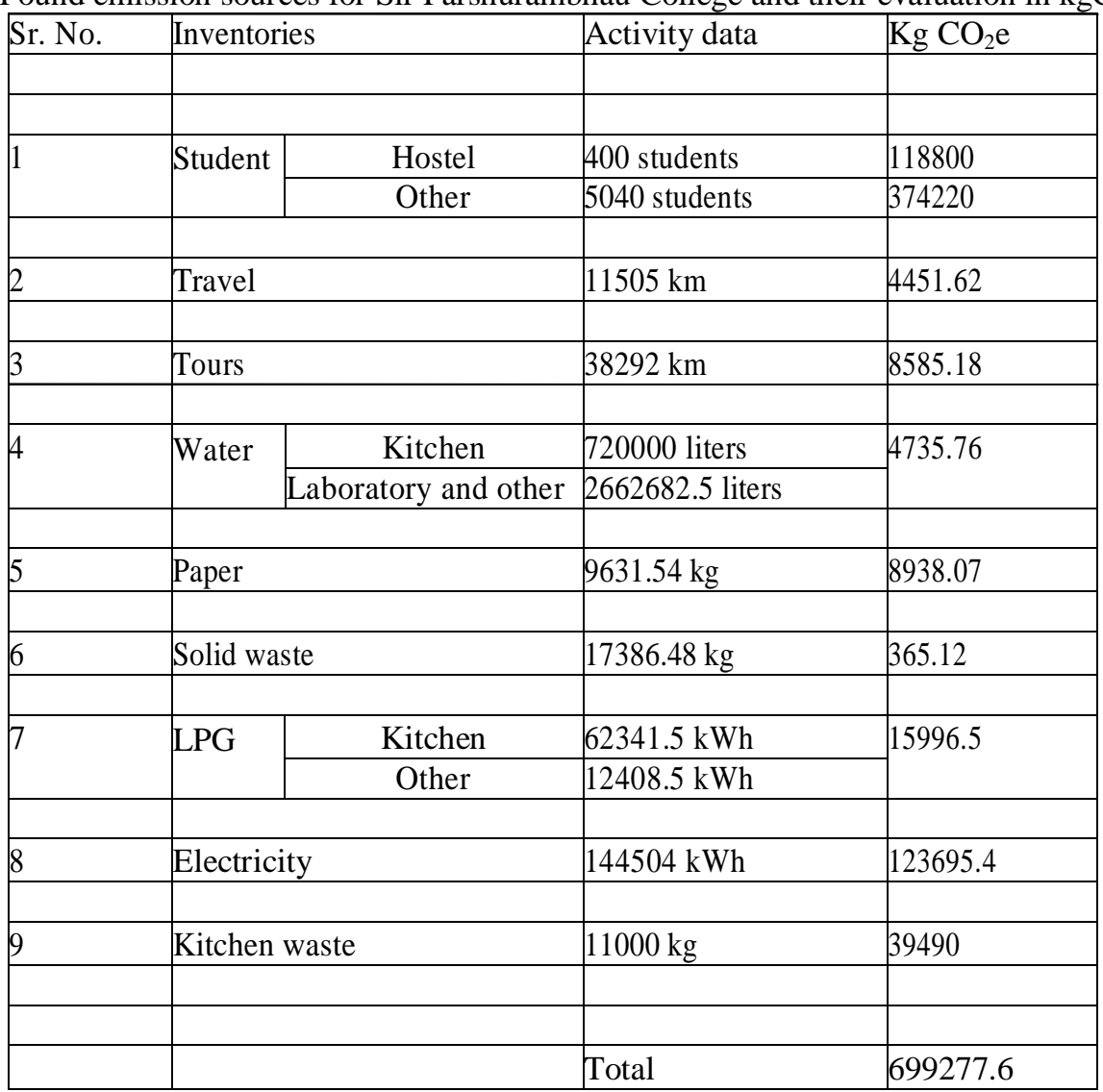


Figure 1: Percent contribution from each source

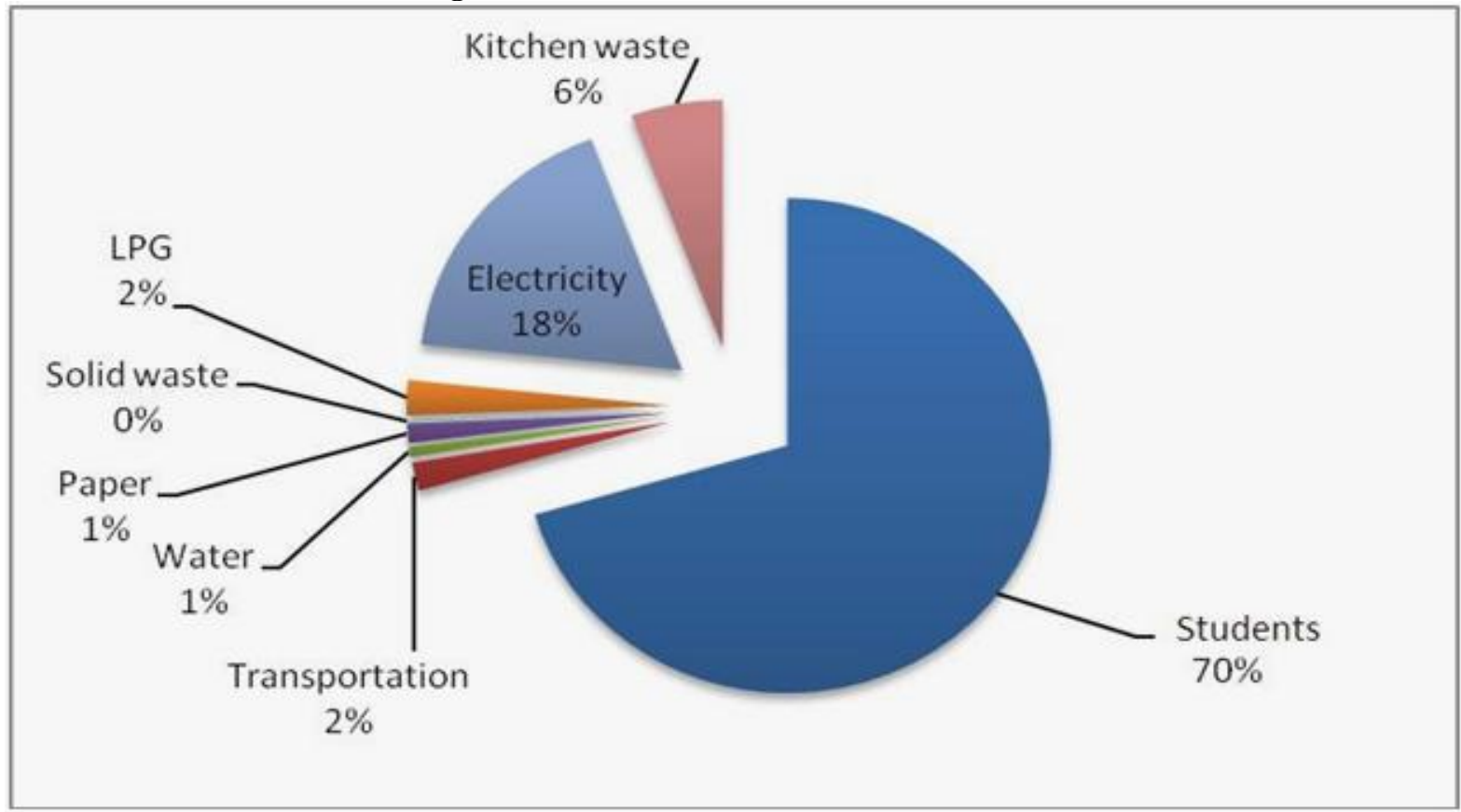

It's found that students contribute almost 70\% of the GHG emissions. This is obvious for a college and also student source (or human source) do not need any mitigation presuming the fact that schools and colleges are building blocks of nation building and hence this emission source must not be taken into consideration for assessment (see Fig. 2). Either way, it's not possible to mitigate GHG emissions through human body.

Figure 2: Percent contribution from each source excluding human factor.

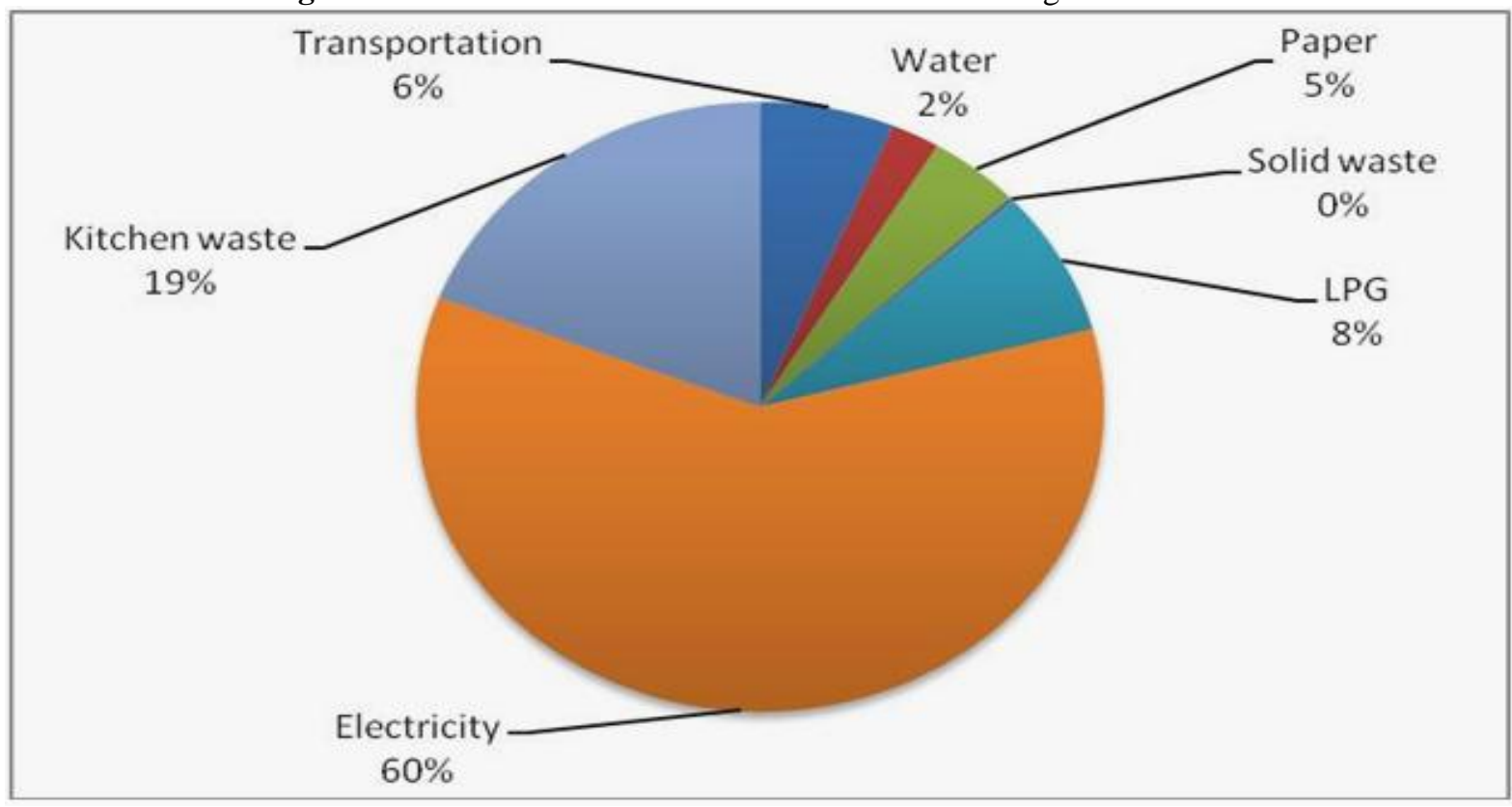

Further, the emissions must be separated into mandatory emissions (M) and waste emissions (W). Separation of emissions is necessary because often unnecessary on site emission sources like garbage waste which includes plastic, papers, etc. which are not responsible are also included in the emissions responsible for the product or services of the functional unit. This adds errors and uncertainties in the evaluation of carbon footprint. It makes the mitigation difficult and the functional unit never knows where to put effort on. But bifurcation and further explained process of evaluation makes the unit aware about where it needs to set mitigation goal and act? Therefore, the separation is very important. 


\subsection{Waste emissions}

Emissions of GHGs which are not related to any special purpose (production or services) of a functional unit are known as waste emissions. For simplicity, they can be called as unnecessary emissions.

Solid waste, kitchen waste, water and LPG used for kitchen are the sources responsible for waste emissions; precisely, they are not necessary for the special purpose of the college. It's found that waste emissions mostly are ready to grave or in the last stage of the lifecycle. This is the reason for all the responsible emissions related to kitchen are expressed separately in Table 2. Waste emissions sum to have $54204.197 \mathrm{kgCO}_{2} \mathrm{e}$ emissions which constitute to be approximately $12.9 \%$ of the total GHG emissions.

For waste emissions, emission density must be evaluated. Emission density is defined as areal density $\left(\mathrm{d}_{\mathrm{W}}\right)$, i.e. the exclusive total waste emissions (W) expressed in mass of $\mathrm{CO}_{2} \mathrm{e}$ per area (A) of the premise.

$$
\begin{aligned}
d_{W} & =\frac{W}{A} \\
& =\frac{54204.197}{101201.568} \\
& =0.536 \mathrm{~kg} / \mathrm{m}^{2}
\end{aligned}
$$

Knowing the emission density, a functional unit can try to decrease its unnecessary emissions every year. Relation to area gives advantage to compare the emission density to that of other functional units. The waste emission density for Sir Parshurambhau College is evaluated to be approximately $0.5 \mathrm{kgCO}_{2} \mathrm{e} / \mathrm{m}^{2}$. Kitchen waste and solid waste are chosen as waste emission sources because neither of the two is necessary for the special purpose of the college.

\subsection{Mandatory emissions}

Emissions of GHGs adding to the carbon footprint which are compulsory or required for the special purpose (production or services) of a functional unit are known as mandatory emissions.

For example; carbon emission by machines while manufacturing products, use of exam papers by a university, official papers, usage of water for the special purpose, etc. Carbon emission by human body is neither mandatory nor waste; it's a source we can't keep control on. Usage of water is unavoidable cause of certain and obvious reasons. Even most of the industrial machines require high amount of water and hence it is treated as mandatory for applicable sources. Mandatory emissions for the college are evaluated to be $152053.4285 \mathrm{kgCO}_{2}$ e emissions (see Table 3) which constitute to be approximately $22 \%$ of the GHG emissions.

Electricity accounts to be major contributor for mandatory GHG emissions and has $81 \%$ contribution. Since electricity and LPG come under scope $2[10,4]$ emissions, we don't have enough control over them. Proper usage of such sources would help minor decrease in the emissions. Small decrement too has equal importance when it comes to GHG mitigation. The sources we can have enough control on are transportation, water and paper.

Work done must be evaluated for GHG emissions from mandatory inventories (because mandatory emissions are the only GHG emissions responsible for a product or a service). Work done is necessary to be evaluated because during the evaluation process it gives the graphical relation between emission intensity VS emission power of the production or service which is important for understanding carbon footprint to the depth. We must therefore evaluate emission intensity and areal contribution for each of the individual inventories further to obtain emission power.

Emission intensity is a measure of total $\mathrm{CO}_{2} \mathrm{e}$ from mandatory inventories (mandatory emissions) per 'n' number of products (or some measure of input or output applicable $[9,18]$ ). 
Figure 3: Percent contribution from each source for mandatory emissions

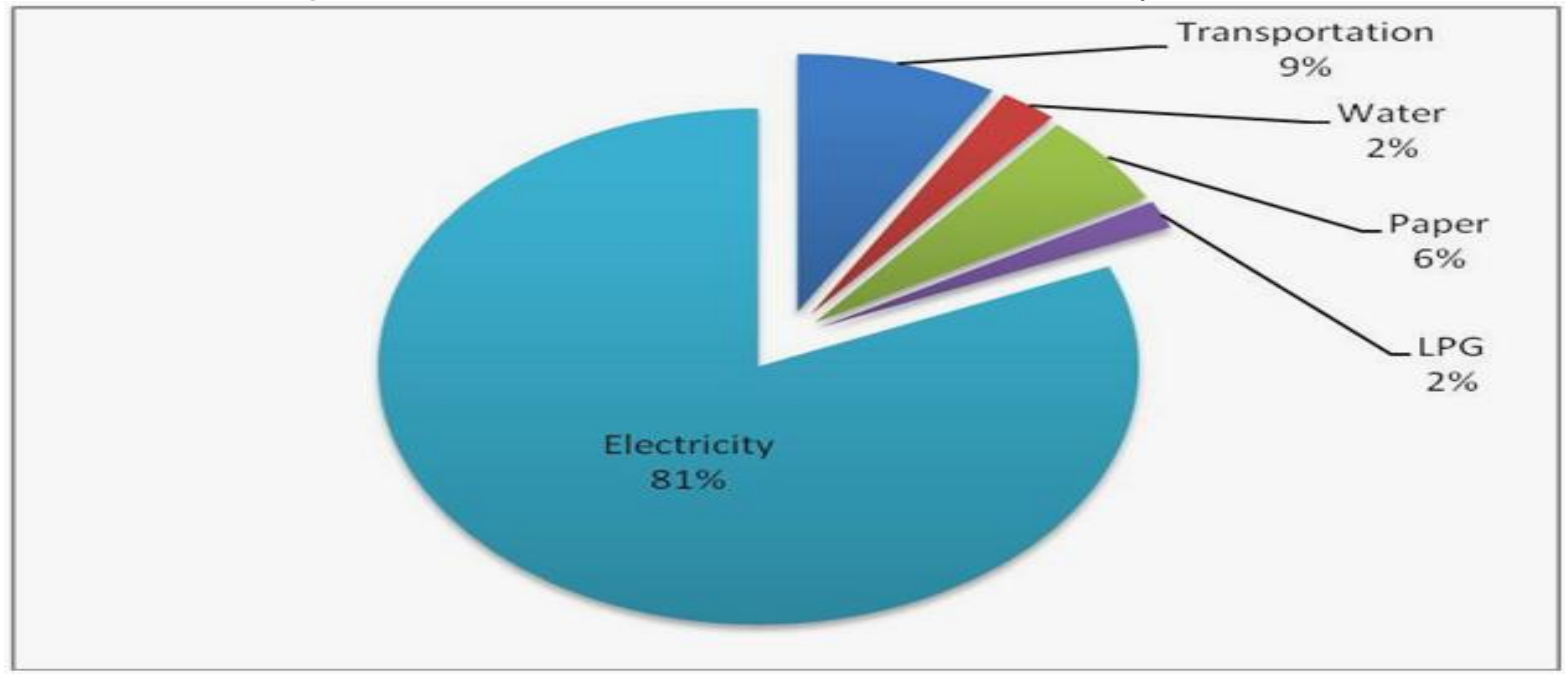

'M' is mass of GHG emissions from mandatory inventories and ' $\mathrm{n}$ ' is total number of products or some other output quantity (in this case, students are considered to be products).

$$
I_{E}=\frac{M}{n}
$$

The total emission intensity is calculated to be $27.9 \mathrm{~W} / \mathrm{m}^{2}$ approximately. For a college or a university, students can be treated as products. Therefore, we have chosen ' $n$ ' to be number of students in the college. If a functional unit produces multiple products, the intensities of individual products must be evaluated and then summated to find the total intensity. Taking the intensity as a whole for multiple products adds errors to the calculation. This is because two groups of different products in a same functional unit may have different emission rates.

Areal contribution $\left(\mathrm{A}_{\mathrm{C}}\right)$ is ratio of mass of $\mathrm{CO}_{2} \mathrm{e}$ from the inventory over total mass of mandatory emissions times area of the premise. 'A' stands for the total area of the premise.

$$
A_{C}=\frac{M}{T_{e}} A
$$

The total area of the premise is $101201.6 \mathrm{~m}^{2}$.

Emission intensity and areal contribution for each of the inventories must be evaluated individually (see Table 3).

Furthermore, emission power must be evaluated (as shown in Table 3) to get the graphical relation between emission intensity and emission power.

Table 3: Evaluation of total work done

\begin{tabular}{|l|l|l|l|l|l|}
\hline $\begin{array}{l}\text { Emission } \\
\text { inventories }\end{array}$ & $\begin{array}{l}\text { Mandatory } \\
\text { emissions }\end{array}$ & $\begin{array}{l}\text { Areal } \\
\text { contribution }\end{array}$ & $\begin{array}{l}\text { Emission } \\
\text { intensity }\end{array}$ & $\begin{array}{l}\text { Emission } \\
\text { power }\end{array}$ & Work done \\
\hline & & & & & \\
\hline & $\mathrm{kgCO}_{2} \mathrm{e}$ & $\mathrm{m}^{2}$ & $\mathrm{~W} / \mathrm{m}^{2}$ & $\mathrm{~J} / \mathrm{year}$ & $\mathrm{kWyear}$ \\
\hline Transport & 13036.774 & 8676.831 & 2.396 & 20793.72 & 20.79 \\
\hline Water & 3727.755 & 2481.066 & 0.685 & 1700.14 & 1.7 \\
\hline Paper & 8938.06 & 5948.867 & 1.643 & 9774.142 & 9.774 \\
\hline LPG & 2655.419 & 1767.35 & 0.488 & 862.696 & 0.862 \\
\hline Electricity & 123695.42 & 82327.446 & 22.738 & 1871972.066 & 1871.972 \\
\hline & & & & & 1905102.784 \\
\hline Total & 152053.428 & 101201.568 & 27.951 & & 1905.102 \\
\hline
\end{tabular}

Emission power is defined as emission intensity times corresponding area for each inventory in mandatory emissions. It's derived from the inverse square law which states, 'intensity is inversely proportional to area and directly proportional to the power'. 


$$
\begin{aligned}
I_{E} & =\frac{P_{E}}{A_{C}}-\text { inverse square law } \\
P_{E} & =A_{C} \times I_{E}
\end{aligned}
$$

The emission power from mandatory emissions is found to be $1905102.784 \mathrm{~J} / \mathrm{year}$ for the college. It is recommended to use year as unit of time because carbon footprint is usually carried out over a period of year. One can evaluate that for several years and represent average emission power or average work done per year.

The graphical relation between emission intensity and emission power is given in Fig. 4 below. The regression error is found to be 1 which means there is no error for the plots to match the power series curve. The equation of the curve obtained, $y=3620.677 \mathrm{x}^{2}$ is parabolic. The general equation for the curve can be written as $\mathrm{y}=\mathrm{ax}^{2}$, this is valid for any assessment data. The coefficient 'a' could be found by dividing $\mathrm{A}_{\mathrm{C}}$ by $\mathrm{I}_{\mathrm{E}}$.

$$
\begin{aligned}
a & =\frac{A_{C}}{I_{E}} \\
& =\frac{101201.568}{27.951} \\
& =3620.677
\end{aligned}
$$

From the emission power, work done can be evaluated.

Work done is defined as emission power of the mandatory emissions times the total time period (t) chosen for an assessment of carbon footprint.

Figure 4: Emission Power VS emission intensity graphical relation

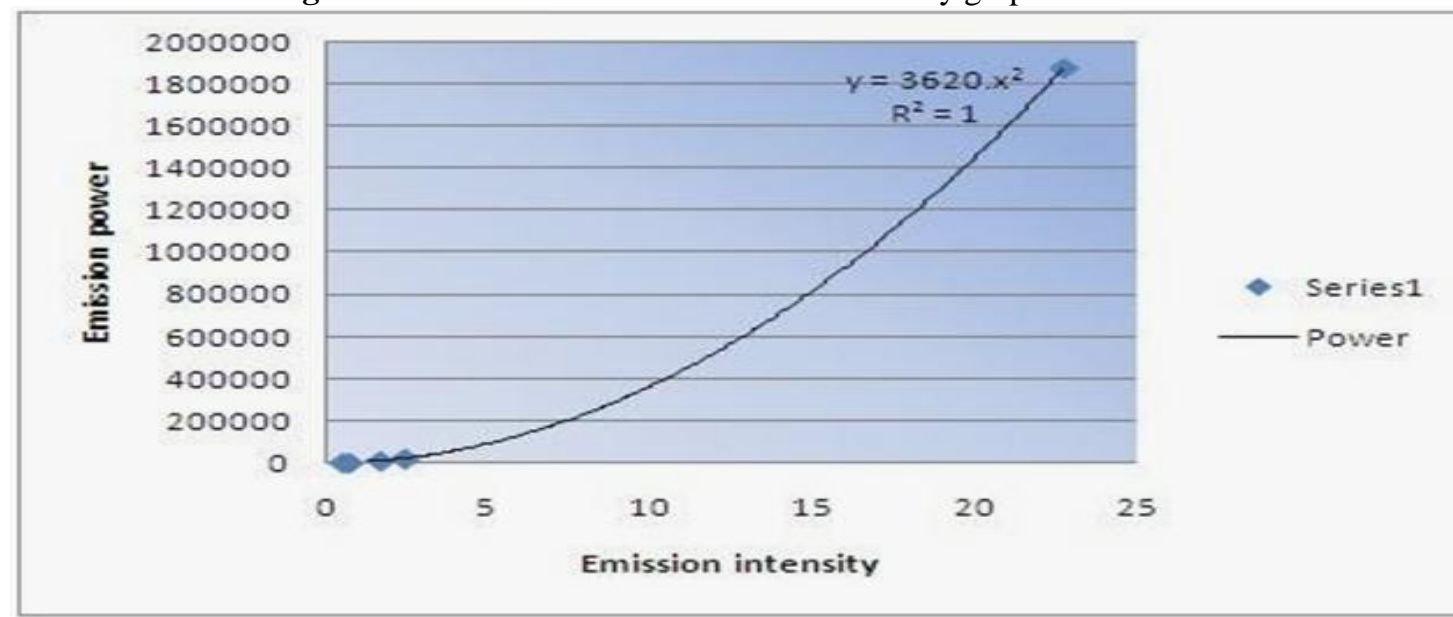

$$
\mathrm{W}=\mathrm{P}_{\mathrm{E}} \mathrm{X}
$$

Work done must be treated as the carbon footprint of any functional unit because it gives more scientific information in joules (converted into kWyear) and involves spatial and temporal system boundaries in itself; thus quantifying the carbon footprint. Also it gives graphical relation between emission intensity and emission power along with the equation of the parabolic curve using the coefficient 'a'. The work done is calculated to be $1905.10 \mathrm{kWyear}$ for the college. This methodology provides various entities for comparison of carbon footprint between different functional units. Although a wider consent is necessary to set a large mitigation goal, institutes, organizations and industries may practice the presented methodology to extract necessary information regarding their carbon footprint. 


\section{Conclusions}

The presented research work provides a broad perspective on further bifurcation of inventories into mandatory emissions and waste emissions. This promotes a more rational idea about how and where a functional unit needs to put effort on in order to reduce its GHG emissions. The equations so derived provide a more rational perspective and give information like emission density, emission intensity, emission power and work done which can be derived from the collected database of the carbon footprint of a functional unit. Work done must be treated as the real carbon footprint. Because each term involved in the evaluation of work done provides important and necessary information for mitigating GHGs and understanding the carbon footprint patterns. Any functional unit can use the presented equations to evaluate carbon footprint and find the parameters to focus on. This makes assessing and offsetting or eliminating GHG emissions easier and also overcomes most of the uncertainties and errors. The work done by the GHG emissions is found to be 1905.10 $\mathrm{kWyear}$ and the equation of power VS intensity curve is found to be $\mathrm{y}=3620 \mathrm{x}^{2}$ for S P College. The methodology, if followed by interrelated functional units, automatically generates a supply chain [5]. There exists no need for considering supply chain tables and having a look at the process, because the methodology itself eliminates double counting of the emissions. This process for evaluation of carbon footprint can be practiced at all scales of functional units. For example, city, industry, organization, institute, local residence etc. A wider perspective is initiated by the methodology along with its preciseness and completeness. Thus, it can be practiced widely to know accurate carbon footprint and set a rightful and scientific mitigation goal. This would help reduce GHG emissions in a rational way.

\section{Acknowledgements}

We especially thank Dr. Dilip N Sheth, principal of S P College, Pune for their cooperation and permitting us to evaluate the carbon footprint of the college. Also, we thank Dr. Sunil Kulkarni for their valuable coordination.

\section{References}

1. Government of India. Ministry of Commerce Industry Department of Industrial Policy Promotion (Manufacturing Policy Section). PRESS NOTE NO. 2 (2011 SERIES)

2. MSME schemes, Government of India. National Institute for Micro, Small and Medium Enterprises (nimsme) (An organisation of the Ministry of MSME, Govt. of India) Yousufguda, Hyderabad 500045

3. Thomas Wiedmann and Jan Minx. A definition of Carbon Footprint. Ecological Economic Research Trends. Nova science publishers .inc. 2007.

4. Dr. Thomas Wiedmann and John Barrett. A Greenhouse Gas Footprint Analysis of UK Central Government, $1990-2008$ http://randd.defra.gov.uk/Default.aspx ? Menu=Menu\&Module=More\&Location=None $\&$ Completed=0 \&ProjectID=17486

5. Carbon footprint estimation for a sustainable improvement of supply chains: state of the art, Pilar Cordero. Universitat Politecnica de Valencia (Spain) http://www.jiem. org/index.php/jiem/article/view/570/491

6. United Nations Framework Convention on Climate Change (UNFCCC)- Kyoto protocol. http://unfccc.int/kyoto_protocol/items/3145.php

7. Carbon gas conversion factor respiratory- DEFRA

8. Thomas Wiedmann and Jan Minx- A definition of carbon footprint. ISAUK Research Consulting, Durham, DH7 7FB, UK (www.isa-research.co.uk); Stockholm Environment Institute, University of York, Heslington, York, YO10 5DD, UK

9. Denny Ellerman and Ian Sue Wing. Absolute vs. Intensity-Based Emission Caps. Joint Program on the Science and Policy of Global Change MIT E40-428. E-mail: glob-alchange@mit.edu

10. Hillary Benn, Ed Milliband. Guidance on how to measure and report your green house gas emissions. Department for Environment, Food and Rural A airs Nobel House.

11. Peter Thatcher, Lancaster. How much does breathing contribute to climate change? Science World

12. Shaleen Agarwal. Final Report Market Survey for Fuel Consumption norms for Diesel Trucks Buses in India. Petroleum conservation research association(PCRA)

13. Glen P Peters. Carbon footprints and embodied carbon at multiple scales. Center for International Climate and Environmental Research Oslo (CICERO), PB 1129 Blindern, Oslo, Norway

14. Tao Gao*, Qing Liu and Jianping Wang. A comparative study of carbon footprint and assessment standards. International journal of low carbon technologies. http: //ijlct.oxfordjournals.org/content/early/2013/06/24/ijlct.ctt041.full

15. Hogne N. Larsen and Edgar G. Hertwich. Implementing Carbon-Footprint-Based Calculation Tools in Municipal Greenhouse Gas Inventories, The Case of Norway. Journal of industrial ecology

16. Weiling Wu. Carbon Footprint a Case Study on the Municipality of Haninge. KTH, Uppsala University, and Swedish University of Agricultural Sciences.

17. Wackernagel and Rees. Our ecological footprint, 1996

18. Rui Zhao, Pauline Deutz, Gareth Neighbour and Michael McGuire. Carbon emissions intensity ratio: an indicator for an improved carbon labeling scheme. IOP science.

19. Aida Sefic Williams. Life Cycle Analysis: A Step by Step Approach. ISTC Reports. Illinois Sustainable Technology Center. 